\title{
Abnormal Proliferation of Gut Mycobiota Contribute to The Aggravation of Type 2 Diabetes
}

\section{Li Bao}

\section{Ying Zhang}

Guo Zhang

De Jiang

Dan Yan ( $\nabla$ danyan@ccmu.edu.cn )

Beijing Friendship Hospital, Capital Medical University

\section{Article}

Keywords: gut mycobiota, insulin resistance, Candida albicans, Dectin-1, 1,3- $\beta$-glucan

Posted Date: March 7th, 2022

DOI: https://doi.org/10.21203/rs.3.rs-1379911/v1

License: (1) This work is licensed under a Creative Commons Attribution 4.0 International License. Read Full License

Version of Record: A version of this preprint was published at Communications Biology on February 28th, 2023. See the published version at https://doi.org/10.1038/s42003-023-04591-x. 


\section{Abstract}

Type 2 diabetes (T2D) constitute a worldwide health threat, and the underlying mechanism for the development and progression of T2D is complex and multifactorial. During the last decade, gut commensal bacteria have been found to play a crucial role in the regulation of T2D and related metabolic disorders. However, as a considerable component in gut microbiome, the relationship between mycobiota and T2D and related metabolic disorders remains unclear. As a proof-of-concept, we observed that the ablation of the commensal fungi in mice can protect HFD induced insulin resistance and related metabolic disorders. Both ITS2 sequencing and culture-dependent analysis show the enrichment of Candida albicans in samples from individuals with T2D (Chinese Clinical Trial Registry, ChiCTR2100042049). Repopulation with $C$. albicans in HFD mice accelerated insulin resistance and related disorders. Mechanically, we found the $\beta$-glucan from $C$. albicans mirrored the deteriorating effect of $C$. albicans through the dectin-1 dependent pathway. Our current findings support that gut mycobiota play an important role in the progress of T2D and indicated the preventing of gut mycobiota is a promising strategy to alleviate insulin resistance and related metabolic dysfunctions.

\section{Introduction}

Type 2 diabetes (T2D) and related metabolic disorders constitute a worldwide health threat ${ }^{1}$, and the underlying mechanism for the development and progression of T2D is complex and multifactorial. During the last decade, gut microbiota-mainly represent by gut commensal bacteria, have been found to play a crucial role in the regulation of energy and substance metabolism, which affect the development of T2D and related metabolic disorders. ${ }^{2-5}$ Imidazole propionates (ImP), produced by several bacterial strains, including Streptococcus mutans and Eggerthella lenta, which was found to be elevated in individuals with T2DM and impairs insulin signaling through activation of the p38y-p62-mTORC1 pathway. ${ }^{6}$ GUDCA, an endogenous bile acid conversed by gut bacteria, was found to be increased by metformin treatment and improve insulin sensitivity though an intestinal FXR dependent pathway. ${ }^{7}$ However, the function and action mechanisms of remain gut symbiont on the host remain unknown.

In recent few years, as a considerable component in gut microbiome, the gut mycobiota (gut fungi) has found to have enormous impact in mammalian health and disease. ${ }^{8-9}$ Increasing evidence suggests that commensal fungi can affect the course and severity of a several of diseases, such as asthma, inflammatory bowel disease, pancreatic carcinoma, and alcoholic liver disease. ${ }^{10-13}$ Candidalysin, a peptide toxin secreted by the commensal gut fungus Candida albicans, was found to enhance alcoholassociated liver disease independent of the $\beta$-glucan receptor CLEC7A, and is also associated with worse clinical outcomes in patients with alcoholic hepatitis.${ }^{14}$ However, the relationship between mycobiota and other diseases, especially in T2D and related metabolic disorders remains unclear.

Herein, we observed that the ablation of the commensal fungi in mice can protect HFD induced insulin resistance and related metabolic disorders, which indicated the potential role of mycobiota in T2D. Both ITS2 sequencing and culture-dependent analysis show the enrichment of $C$. albicans in samples from 
individuals with T2D. Repopulation with $C$. albicans in HFD mice accelerated insulin resistance and related disorders. Mechanically, we found the $\beta$-glucan from $C$. albicans mirrored the deteriorating effect of $C$. albicans through the dectin-1 dependent pathway. Furthermore, $C$. albicans also interact with gut bacteria, with the enrichment of Staphylococcus xylosus and Mucispirillum schaedieri. Our current findings support that gut mycobiota play an important role in the progress of T2D and indicated the preventing of gut mycobiota is a promising strategy to alleviate T2D and related metabolic dysfunctions.

\section{Results}

\section{Eliminate of gut mycobiota alleviated insulin resistance and related metabolic disorders in HFD-fed mice}

To evaluated weather gut mycobiota play a role in the occurrence and progress of T2D and related metabolic disorders, HFD-fed mice treated with or without the antifungal agent amphotericin B (AmB) were performed to determine if eliminate of the intestinal fungal community impacts the progress of insulin resistance and related disorders. AmB is an ideal agent for the clear of gut mycobiota due to its broad-spectrum anti-fungi activity, low permeability, and low bioavailability, thus may confined the effects in the intestinal lumen. ${ }^{15-16}$

Sequential monitoring of the blood glucose showed that AmB improved fasted blood glucose and freediet blood glucose significantly (Fig. 1A-B). Insulin resistance is a central pathological factor in T2D and a causal link between T2D and related metabolic disorders, including obesity, hyperlipidemia, and nonalcoholic fatty liver disease (NAFLD) ${ }^{17}$ HFD mice displayed apparent insulin resistance (Fig. 1C-G). AmB treatment significantly decreased the HOMA-IR (Fig. 1C). Moreover, in the oral glucose tolerance test (OGTT), HFD mice treated by AmB showed decrease of blood glucose levels at 40 and 80 min compared with the HFD mice (Fig. 1D). The AUC of the AmB group were much lower than that in the HFD group (Fig. 1E). Above insulin sensitizing effects also proved by ITT test (Fig. 1F, G), which together indicated the role of gut intestinal fungal community in the control of blood glucose level and insulin resistance.

Obesity, dyslipidemia, and NALFD is the most common T2D related disorders with severely impair on human health. Compared to the vehicle-treated HFD-mice, supplementation with AmB significantly prevented body weight gain at 2-week treatment, and extend to the end of treatment (Fig. $1 \mathrm{H}$ ). Regarding to the blood dyslipidemia, AmB treated HFD-mice effectively improved blood dyslipidemia by reducing the concentrations of plasma total cholesterol (TC), low-density lipoprotein cholesterol (LDL-C), and triglycerides (TGs) (Fig. 1I, J). Moreover, the size of adipocytes in the white adipose tissue (WAT) of AmB treated HFD-mice was reduced (Fig. 1K).

In terms of NAFLD, we noticed the recovery of liver weight to body weight ratio treated by AmB (Fig. 1L) and the improvement of markers of hepatocellular injury, such as plasma alanine aminotransferase (ALT) and aspartate aminotransferase (AST; Fig. 1M). In addition, AmB treated HFD-mice apparently reduced hepatic macrosteatosis, hepatocyte ballooning, and fat deposition, as indicated by liver sections HE and 
Oil 0 red staining and microscopy observation (Fig. 1N, 0). Furthermore, AmB supplement reverse systems inflammation, a causal link between insulin resistance, obesity and diabetes, as indicated by the alleviated plasma TNF-a and LPS level (Fig. 1P, Q).

Overall, our results found that the gut mycobiota eliminate treated by AmB have profound impact on T2D, insulin resistance and related disorders for the first time, indicated that gut mycobiota may play an important role in the progress on T2D and related disorders.

\section{Gut Mycobiota Alternations In T2d Patients}

For further investigate the relationship between gut mycobiota and T2D, we collected stool samples from 11 individuals with type 2 diabetes and 6 healthy volunteers. The over-growth of mycobiota in T2D group was observed by real-time RT-PCR (Fig. 2A). Further, we performed ITS2 sequencing to explore the composition and abundance of gut mycobiota between T2D and control group. There is no significant difference between two groups for a diversity, which indicated by OTU number and Shannon index (Fig. 2B, C). The $\beta$ diversity show the composition and abundance of microbiota from T2D group was significantly differed from that of control group (NMDS plot, MRPP test, $p<0.001$; Fig. 2D).

Among the fungal taxa, the phylum Ascomycota dominated the mycobiota, while Basidiomycota was observed as the second most abundant phylum (Fig. 2E) in both T2D and control groups. Additional differences were observed at lower taxonomic levels, at the genus level, 6 fungal features were enriched in T2D, including Candida, Aspergillus, Sarocladium, Debaryomyces, Kurtzmaniella and Monascus, whereas the genus including Fusarium and Cladosporium were significantly reduced in T2D patient (Fig. 2F). The analysis with linear discriminant analysis (LDA) effect size (LEfSe) method revealed a significant increase in Candida albicans, Saccharomycetales in T2D patient, accompanying the decrease of Hypocreales, Sordariomycetes, and Nectriaceae (Fig. 2G, H).

While sequencing-based profiling is useful for providing a snapshot of fungal DNA, whether the associated organisms are passive bystanders dead upon arrival or viable inhabitants of a given niche cannot be reliably determined. We aimed, therefore, to determine if the identified sequences came from viable organisms via cultivation, and if so, we hypothesized that the genomic and functional characteristics of these organisms could provide insights into the micro-environment of gut mycobiota. In according with sequencing results, we discovered a significant enrichment of Candida albicans in the T2D group (Fig. 2I, Fig. S1), which indicated $C$. albicans as a potential pathogens in the pathological process of insulin resistance and diabetes.

\section{C. albicans accelerated the progress of T2D and related disorders.}

Candida albicans, a prominent opportunistic pathogen responsible for mucosal infection in human immunodeficiency virus-infected patients and nosocomial systemic infections, is now regarded as common gut symbionts colonize all segments of the human digestive tract. ${ }^{18} \mathrm{C}$. albicans is found to 
increase in patients with alcoholic hepatitis and is the most abundant Candida species in patients with end-stage liver disease due to alcohol abuse. ${ }^{19}$ However, the precise role of $C$. albicans in T2D and related disorders remains unknown. Thus, we repopulated HFD-mice with C. albicans, in order to explore the effects of $C$. albicans in insulin resistance and related metabolic disorders.

In comparison with the vehicle group, $C$. albicans significantly accelerated the features of T2D and insulin resistance in the HFD-fed mice, indicated by the increasing fasted blood glucose, HbA1c level and HOMAIR (Fig. 3A-D), and the impairing insulin tolerance and glucose tolerance (Fig. 3E-H). There has no significant change on body weight, liver/body weight index, and plasma lipid profiles (Fig. S2) during $C$. albicans colonization. However, the H\&E and Oil $\mathrm{O}$ red staining also proved the aggravating macrosteatosis and hepatocyte ballooning in C. albicans group (Fig. $3 \mathrm{l}$ ).

\section{The structural characterization of $\beta$ - glucan from C. albicans (CAG)}

We next explored the molecular basis of the insulin resistance inducing effect in C. albicans. 1,3- $\beta$ glucans-a cell wall polysaccharides found in and released from most fungi, including species of Candida, Aspergillus, and Pneumocystis, ${ }^{20}$ were found to make a profound impact on host, including immune response, hepatic injury, carcinogenesis . ${ }^{12-13,21}$ Accordingly, patients and mice with T2D has a higher level of 1,3- $\beta$-glucans in both fecal and plasma (Fig. 4A, B), indicated the potential role of 1,3- $\beta$-glucans on the occurrence of T2D.

Due to the species variety, chemical structure and bio-function of 1, 3- $\beta$-glucans may be widely divergent. For example, the 1, 3- $\beta$-glucans derived from Aspergillus spp. may induce strong immune response and trigger asthma, ${ }^{10}$ on the contrary, the 1,3- $\beta$-glucans isolated from medical fungi Poria cocos exhibit a protective effect on metabolic syndrome. ${ }^{22-23}$ In order to explore the effect of $\beta$-glucan from $C$. albicans (CAG) on insulin resistance and related metabolic disorders, we next identify CAG using a $C$. albicans strain isolated from T2D patient. High-performance size exclusion chromatography analysis of CAG indicated a single homogeneous composition (Fig. 4C). The Fourier transform infrared spectrum (FT-IR) spectrum of CAG showed the absorptions at $3373,2922,1200-1000 \mathrm{~cm}^{-1}$, corresponding to the vibration of $\mathrm{O}-\mathrm{H}, \mathrm{C}-\mathrm{H}, \mathrm{C}-\mathrm{O}, \mathrm{C}-\mathrm{O}-\mathrm{C}$ bonds, respectively. The absorptions at 1074 and $891 \mathrm{~cm}^{-1}$ suggested a pyranose form of sugars with $\beta$ anomeric configuration (Fig. 4D). The monosaccharide composition of the polysaccharide was determined as D-glucose by acid hydrolysis followed with HPLC analysis of the corresponding derivatives (Fig. 4E). By comparing ${ }^{13} \mathrm{C}$ NMR spectra of CAG with the corresponding compounds in the literatures, ${ }^{24}$ confirmed the main chain of CAG to be 1,3- $\beta$-D-glucan on the basis of the signals observed at 73.3 (C-2), 86.7(C-3), 76.8 (C-5) (Fig. 4F-G).

\section{CAG mirrored the pathogenic effect of $\mathrm{C}$. albicans}

As expected, oral gavage of CAG significantly increased both body weight and free diet blood glucose in HFD mice (Fig. 5A, B). HbA1c was rised substantially (Fig. 5C). CAG also deteriorate glucose tolerance and insulin resistance. The AUC during the OGTT and the ITT of CAG-treated HFD mice was much higher 
than that of the vehicle-treated HFD mice (Fig. 5D-G). CAG treatment also caused elevation in the HOMAIR (Fig. $5 \mathrm{H}$ ). The H\&E and Oil $\mathrm{O}$ red staining of liver in CAG group also mirrored the aggravating effect on macrosteatosis and hepatocyte ballooning in $\mathrm{C}$. albicans group (Fig. $5 \mathrm{l}-\mathrm{K}$ ). Above observations indicated that oral CAG exacerbate insulin resistance and related disorders, mirrored the pathogenic effect of $C$. albicans.

\section{CAG induce insulin resistance though the activation of dectin-1 pathway}

Dectin-1 (also known as C-type lectin domain family 7 member $A, C L E C 7 A$ ) is a pattern recognition receptor, which recognizes a variety of 1,3 - $\beta$-glucans. ${ }^{25}$ Upon ligand binding, Dectin-1-Syk pathway activates macrophage polarization by activating IRF5, a transcription factor that regulates proinflammatory $\mathrm{CD} 11 \mathrm{c}^{+}$macrophage differentiation, ${ }^{26}$ which induce insulin resistance and related disorders. CAG treatment significantly induced the expression of genes including Dectin-1, Syk, and Irf5 (Fig. 6B, D), indicate the activation of Dectin-1-Syk pathway. Accordingly, the activation of Dectin-1 signing also observed in the $C$. albicans treated HFD mice (Fig. 6A, C).

The induced dectin-1 pathway is in line with the damaged intestinal barrier function and intestinal inflammation in both CAG and $C$. albicans treated group, compare with vehicle mice respectively, indicated by the reduced Z01 and Ocludin expression (Fig. 6E-F), and the induced inflammatory factors (Fig. 6G-H). Above observation suggested that $C$. albicans and CAG both induced the Dectin-1-Syk pathway through the secretion of 1,3- $\beta$-glucans, thus aggravate the insulin resistance and related disorders.

Furthermore, we performed the dectin- 1 antagonist laminarin to further verify the role of dectin-1 pathway in $C$. albicans CAG induced phenotype, as a dectin-1 antagonist, laminarin treatment reversed the deteriorated effect of CAG on HFD-treated mice, as indicated by body weight gain, fasted blood glucose, ITT, OGTT, HOMA-IR, HbA1c (Fig. 7A-H). In addition, the markers of low-grade systemic inflammation induced by CAG colonization, Including IL-1 $\beta$ and TNF-a, were counteracted by laminarin gavage effectively (Fig. 7l). Thus, we concluded that $C$. albicans induce insulin resistance and related inflammation by a dectin-1 dependent pathway.

\section{The bacterial community change induced by $\mathrm{C}$. albicans and CAG}

Many observations suggest that bacteria and fungi interact within the gut, influencing each other through different levels of symbiosis. ${ }^{27}$ To understand the potential interplay among the interaction between $C$. albicans and differentially abundant bacteria, we performed additional bacterial abundance analysis and estimated the ecological network. The 16S rRNA amplicon profiles showed that the $C$. albicans inoculated mice exhibited an unchanged $a$-diversity but a significant distinction on $\beta$-diversity compared with vehicle treatment group, which reflected by Shannon index and PCoA assay (Fig. 8A, B).

C. albicans treatment enriched the relative abundance of the Phylum Firmicutes, while decrease Phylum Bacteroidetes (Fig. 8C), thus show a remarkable increase on F/B radio, an index partly reflected the 
damaged glucose and lipid metabolism (Fig. 8D). ${ }^{28-30}$ In term of the genus level, Staphylococcus, Mucispirillum is increased by $C$. albicans treatment, whereas the genus Akkermansia is suppressed (Fig. 8E). The analysis with linear discriminant analysis (LDA) effect size (LEfSe) method also revealed the increase in Staphylococcus xylosus and Mucispirillum schaedieri (Fig. 8F, G). A. Muciniphila is an important gut symbiont for the maintenance of metabolic homeostasis and is currently recommended as a new probiotic to cope with obesity, diabetes, and liver diseases in clinics. ${ }^{31}$ On the other hand, the inoculation of $\mathrm{C}$. albicans can promote the growth of Staphylococcus spp. by the formation of polymicrobial biofilm, ${ }^{32}$ thus may further promotes low-grade elevation in plasma LPS (Fig. 8H), a major cause for the initiation of obesity and obesity-related dysfunctions. ${ }^{33}$ Above bacterial dysbiosis induced by $C$. albicans may further explain the disordered insulin sensitivity and glucolipid metabolism.

\section{Discussions}

Current studies indicated that mycobiota are inseparable from human health. Dysbiosis and invasion of mycobiota are confirmed to cause disease in different parts of the body. ${ }^{34}$ The role of fungi in intestinal inflammation and many gastrointestinal and liver diseases have come up. $C$. albicans can worsen colitis in mice and has an increased abundance in patients with Crohn's disease, although the pathogenic mechanisms are not yet fully understood. ${ }^{35}$ Aykut et al. show that fungi migrate from the gut lumen to the pancreas, and that this is implicated in the pathogenesis of pancreatic ductal adenocarcinoma (PDA) ${ }^{36}$ In a study of patients with alcoholic liver disease (ALD), gut fungal load, primarily Candida spp., was increased compared with healthy control individuals. ${ }^{20}$ However, the role of mycobiota in diabetes and insulin resistance has not been clearly implicated. In current prove-of-concept study, by eliminate the commensal fungi in mice, we observed the potential role the mycobiota in diabetes and insulin resistance, which raising the pathogenic potential of misadjusted mycobiota in diabetes and related metabolic disorders.

Candida albicans is a prominent opportunistic pathogen and now regarded as a common gut symbionts colonize all segments of the human digestive tract. ${ }^{18}$ Patients with alcohol use disorder have higher intestinal proportions of $C$. albicans. Candidalysin, a cytotoxic peptide secreted by $C$. albicans, causes direct hepatocyte damage and exacerbates ethanol-induced liver disease in mice. ${ }^{14}$ Otherwise, commensal $C$. albicans also play a protective role in several special situation. Shao et al. show commensal $C$. albicans uniquely activates circulating immune cells to protect against systemic infection by invasive extracellular microbial pathogens. ${ }^{37}$ Thus, the role of commensal C. albicans in diabetes need be established. In current study, combine with high-throughput sequencing and fungal cultivation of clinical sapmles, single fungi gavage and pathway analysis, we conclude that the $\beta$-glucan from common symbiotic fungus $C$. albicans accelerated the progress of T2D and related disorders though the activation of dectin-1 pathway. Above evidence revealed the pathogenic role of $C$. albicans in diabetes and also highlight this microbe as a novel target for the clinical treatment of T2D; that is, developing a 'drugs for bugs' approach in the management of this common disease. 
Many observations suggest that bacteria and fungi interact within the gut, influencing each other through different levels of symbiosis. However, most of the data on fungi-bacteria interactions have been collected in other settings, such as the lung and oral or vaginal cavities. ${ }^{27}$ By the $16 \mathrm{~S}$ rRNA amplicon profiles, we showed that the $C$. albicans gavage enhance the relative abundance of genus Staphylococcus, Mucispirillum and decrease genus Akkermansia. Staphylococcus spp. may produce mounts of LPS, a cell wall component of Gram-negative intestinal bacteria, can enter the blood stream through the impaired barrier of the gut mucosa, thus induce chronic low-grade inflammation (CLGI), a key mediator of the development of obesity, insulin resistance, and diabetes. ${ }^{38-40}$ By the formation of polymicrobial biofilm, increased C. albicans can promote the growth of Staphylococcus spp., thus enrich plasma LPS concentration and aggravate insulin resistance. Akkermansia Muciniphila, a bacterium widely present in the intestinal tract of humans and other animals, is regarded as a core functional microbe with prominent beneficial effects in lipid metabolism disorders and many other diseases. ${ }^{41-43}$ The increased $A$. Muciniphila induced by $C$. albicans indicated the potential antagonism, which may explain the extensive role of commensal $C$. albicans in host physiological status. Further studies need carry out to explore the underlying mechanism between $A$. Muciniphila and $C$. albicans.

In conclusion, this is a prove-of-concept study to explore the role of mycobiota in diabetes and related metabolic disorders. By the ablation of the commensal fungi in mice, we observed the protection of HFD induced insulin resistance. Both ITS2 sequencing and culture-dependent analysis show the enrichment of C. albicans in samples from individuals with newly diagnosed T2D. Repopulation with C. albicans in HFD mice accelerated T2D and related disorders. Furthermore, we found the $\beta$-glucan from $C$. albicans mirrored the deteriorating effect of $C$. albicans through the dectin-1 dependent pathway. $C$. albicans also interact with gut bacteria, with the enrichment of Staphylococcus xylosus and Mucispirillum schaedieri. Our current findings support that gut mycobiota play an important role in the progress of T2D and indicated that the improvement of $C$. albicans is a promising strategy to alleviate T2D and related metabolic dysfunctions.

\section{Materials And Methods}

Animal care and Experiments. All animal experiments were approved by the Ethics Committee of Beijing Shijitan Hospital, Capital Medical University (permission No. 2017-keyanlunshen-35). C57BL/6J mice were purchased from Beijing Vital River Laboratory Animal Technology Co., Ltd. (Beijing, China).

For antifungal treatment assay on HFD-fed mice, 6-week-old C57BL/6J male mice fed with a high-fat diet (60 kcal\% fat, $20 \mathrm{kcal} \%$ proteins, and $20 \mathrm{kcal} \%$ carbohydrates, Cat. D12492i, Research Diet, New Brunswick, NJ, USA). Mice were divided into 3 groups $(n=10)$ randomly: normal diet (Lean-WT) and highfat diet (DIO-WT) were treated with an oral gavage of an equivalent volume of distilled water, and nonabsorbable antifungal drugs amphotericin B (DIO-AmB, $60 \mathrm{mg} / \mathrm{kg} / \mathrm{d}$ ) was orally administrated for 7 weeks. 
For $C$. albicans treatment assay, $C$. albicans isolated from T2DM patients were cultivated on Yeast peptone dextrose Agar (YPD) and potato dextrose agar (PDA, Table 4S) for 5-7 days and then propagated in potato dextrose broth (both at $28^{\circ} \mathrm{C}$ ) prior to oral gavage into mice. To prove the effect of C. albicans (CA), C57BL/ 6 male mice were sorted into 2 groups: DIO-WT (HFD diet + potato dextrose broth), DIO-CA (HFD diet + oral administration of $C$. albicans at the dose of $2 * 10^{7} /$ day for the following 7 weeks).

To prove the effect of 1,3- $\beta$-glucan on HFD-induced T2DM, C57BL/ 6 male mice were sorted into 2 groups: DIO-WT (HFD diet + sterilized water), DIO-Glucan (HFD diet + oral administration of 1,3- $\beta$-glucan at the dose of $100 \mathrm{mg} / \mathrm{kg} / \mathrm{day}$ for the following 7 weeks).

To test whether the aggravation of $C$. albicans on T2DM is depended on dectin-1, dectin-1 antagonist laminarin (Lam) was used. C57BL/ 6 male mice were sorted into 3 groups: DIO-WT (HFD diet + sterilized water), DIO-Glucan (HFD diet + oral administration of 1,3- $\beta$-glucan at the dose of $100 \mathrm{mg} / \mathrm{kg} / \mathrm{day}$ for the

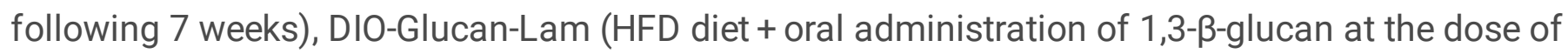
$100 \mathrm{mg} / \mathrm{kg} /$ day and Laminarin at the dose of $250 \mathrm{mg} / \mathrm{kg} /$ day for the following 7 weeks).

Tissue sampling. After treatment, animals were anesthetized with diethyl ether and blood was sampled from the portal and cava veins. After exsanguination, mice were euthanized by cervical dislocation. Abdominal white adipose tissue (WAT), intestines, and liver were precisely dissected, weighed, immediately immersed in liquid nitrogen, and stored at $-80^{\circ} \mathrm{C}$ for further analysis.

Biochemical analyses. The levels of serum glucose, serum insulin, triglyceride (TG), and total cholesterol (TC), high density lipoprotein cholesterol (HDL-C), low density lipoprotein cholesterol (LDL-C), and glycated hemoglobin A1C (HbA1C) were

measured as previously described. ${ }^{44}$ The homeostasis model assessment of

insulin resistance (HOMA-IR) formula was as follows: HOMA-IR = serum glucose levels $(\mathrm{mmol} / \mathrm{I})$ * insulin levels $(\mathrm{mU} / \mathrm{l})$ / 22.5. Serum TNFa, IL-6, IL-1 $\beta$ and IL-10 levels were quantified using TNFa, IL-6, IL-1 $\beta$ and IL-10 enzyme-linked immunosorbent assay (ELISA) kit (CUSABIO, Wuhan, China) following the manufacturer's instructions.

Insulin Tolerance Test (ITT) and Oral Glucose Tolerance Test (OGTT). ITT was performed by injecting insulin $(0.8 \mathrm{U} / \mathrm{kg})$ intraperitoneally after a $4 \mathrm{~h}$ fasting. OGTT was performed by oral administration of Dglucose $(2 \mathrm{~g} / \mathrm{kg})$ after overnight fasting. The level of blood glucose was measured using a glucose meter (AccuCheck, Roche, Switzerland) before insulin or glucose load ( $0 \mathrm{~min})$ and at 40, 80, and $120 \mathrm{~min}$ after insulin or glucose load. The area under the curve (AUCs) generated from the data collected during the ITT or OGTT were calculated by GraphPad 7.0.

Histopathological Examination. Samples of liver and WAT were resected and fixed with $10 \%$ formaldehyde phosphate buffered saline ( $\mathrm{pH} 7.4)$, then embedded in paraffin, sectioned, stained with 
hematoxylin/eosin (H\&E stain), Oil Red O, and finally analyzed by light microscopy (Nikon Eclipse E200, Nikon, Japan).

Real-time qPCR analysis. Total RNA was extracted from the intestinal tissue with the TRIzol reagent according to the manufacturer's protocol (Invitrogen, Carlsbad, CA, USA), Quantification analysis of total RNA were performed by running $1 \mathrm{uL}$ of sample on an Agilent 2100 bioanalyzer (Agilent RNA 6000 Nano kit, Agilent). Reverse transcription was performed on $2 \mu \mathrm{g}$ of total RNA using a cloned AMV first-strand cDNA synthesis kit (Tiangen, Beijing, China). Primers used for CDNA amplification by real-time PCR are listed in Table 3S.

Glyceraldehyde-3-phosphate dehydrogenase (GAPDH) was used as the housekeeping gene for normalization of the target genes expression. PCR reactions were performed using KAPA SYBR FAST Qpcr Master Mix 2* kit (KK4601-07959389001) using a QuantStudio 6 Flex Real-Time PCR Systems.

Western blotting. Colon protein was extracted using RIPA lysis buffer (G2002; Servicebio) and PMSF (G2008; Beyotime). The protein amount was measured using a BCA protein assay reagent (P0012; Beyotime). Equal amounts of proteins were boiled in SDS-PAGE sample loading buffer (P0015L; Beyotime) for $15 \mathrm{~min}$ and then separated on a 10\% SDS-PAGE gel and transferred onto a PVDF membrane (IPVH00010; Merck Millipore Ltd.). Membranes were soaked with 5\% nonfat milk in TBST (20 $\mathrm{mM}$ Tris $\mathrm{Cl}, 150 \mathrm{mM} \mathrm{NaCl}$, and $0.05 \%$ Tween-20, $\mathrm{pH} \mathrm{7.4)} \mathrm{for} 1 \mathrm{~h}$ at room temperature. Subsequently, membranes were incubated with primary antibodies at $4^{\circ} \mathrm{C}$ overnight, followed by incubation with Alexa Fluor 790 goat anti-rabbit IgG H\&L (1:10000; ab186697; Abcam, Cambridge, UK) or Alexa Fluor 680 goat anti-mouse IgG (1:10000; ab186694; Abcam). Finally, fluorescent signals were collected using an Odyssey infrared imaging system (LI-COR, Lincoln, NE). The intensities of protein bands were quantified with the Photoshop software (Adobe Systems, San Jose, CA), and the values were normalized to $\beta$ tubulin. Primary antibodies against DECTIN-1 (GB111816; 1:500) were purchased from Servicebio.

Human serum and fecal samples. Twenty-six T2DM patients and fifteen healthy individuals without chronic disease were included in our experiment, and we obtained blood from the vein for the $\beta$-glucan assay. Stool samples from 11 T2DM patients and 6 healthy individuals without chronic disease were collected (Table 1S), and DNA was extracted from fecal samples for the fungal microbiome. The study was approved by the Ethics Committee of Beijing Shijitan Hospital Affiliated to Capital Medical University (2017-035) and registered on the Chinese Clinical Trial Registry (ChiCTR2100042049), as well as conducted under the guidelines of the Helsinki Declaration.

Microbial community analysis. (i) Fungi. The fungal microbiome was analyzed using high throughput sequencing technology. Over 83,234 paired-reads were collected from ITS2 regions using Illumina Novaseq platform. Of these, 65,181 reads with high quality sequences were selected for the later analysis. The reads were assigned to operational taxonomic units (OTUs) with a $97 \%$ similarity threshold and taxonomy assignment of the resulting OTU was carried out using the BLAST against the UNITE reference database. 
The total fungi and the total $C$. albicans were assayed by a two-step nested PCR method for amplification as previously described. ${ }^{23}$ Firstly, ITS fragments were amplified with the primers ITS1 and ITS4 using a KAPA HiFi HosStart Ready Mix (Kapa Biosciences, Wimington, MA), then the amplicon was performed for 2nd PCR using the primers $1737 \mathrm{~F}$ and 2043R (Table 3S).

(ii) Bacteria. Total DNA was isolated from cecum content, sequencing the variable V3 and V4 regions of the 16S rRNA gene was performed with the Illumina NovaSeq platform. The 16S rRNA gene V3-V4 region was amplified using the primers 341F (CCTAYGGGRBGCASCAG) and 806R

(GGACTACNNGGGTATCTAAT). After shear filtration of reads, an average of 82,403 reads was measured per sample. A total of 52,991 effective data were obtained through quality control with the effective rate of $62.99 \%$. The reads were assigned to operational taxonomic units (OTUs) with a $97 \%$ similarity threshold, and a total of 605 OTUs were annotated to species levels with silva132 databases. Data were analyzed as described in our early report. ${ }^{44}$

Culture of fungi. Gut fungi in human fecal sample were cultured using a described method. ${ }^{45} 100 \mathrm{mg}$ of fecal samples from 6 healthy individuals and 11 T2D patients were diluted with $900 \mu \mathrm{L}$ of phosphate buffer saline (PBS) for solid fungal culture. $50 \mu \mathrm{L} 10$-fold dilutions was used for culturing on Dixon agar (DIX) and PDA solid culture media supplemented with 3 antibiotics; namely, colistin ( $30 \mathrm{mg} / \mathrm{L}$ ), vancomycin (30 mg/ L) and imipenem (30 mg/ L) (Table 4S).

C. albicans strains were grown in antibiotics and incubated at $30^{\circ} \mathrm{C}$ with shaking at $150 \mathrm{rpm}$ for $24 \mathrm{~h}$. Cell density was analyzed by measuring optical density at $600 \mathrm{~nm}$ in a microplate reader (Molecular Devices). Cell from YPD broth (Table 4S) were collected by centrifugation, washed twice with PBS and adjusted to the required cell density.

\section{Molecular Fungal Identification}

Direct ITS analysis was performed for fungal species isolates. The ITS1 (TCCGTAGGTGAACCTGCGG) and ITS4 (TCCTCCGCTTATTGATATGC) primers were used for PCR and sequencing. The fungi were identified on the basis of morphology and the DNA sequences of the ITS regions of their ribosomal RNA gene. Analysis of sequences was perfomed using BLAST in GenBank, and the results were seen in Table 2S.

\section{Preparation and analysis of $1,3-\beta-$ glucan isolated from $C$. albicans}

1,3- $\beta$-glucan were isolated from the above mentioned $C$. albicans as previous reported (CAG) with modification. ${ }^{46}$ The levels of CAG was measured with 1,3- $\beta$-DG ELISA kit according to manufacturer's instructions. The structural characteristics of CAG were analysis by HPGPC, IR and NMR. IR data was obtained on a Nicolet IS5FTIR spectrophotometer using the KBr-disk method. NMR spectra were acquired with Bruker Avance-500 spectrometer and CAG was dissolved in DMSO- $d_{6}$. HPLC using pre-column 
derivatization with 1-phenyl-3-methyl-5-pyrazolone (PMP) was used to analyse mono-saccharides as previous described with minor revise. ${ }^{22}$

Statistical analysis. All results are expressed as mean \pm SEM. For multiple comparisons, statistical analysis was performed using one way or two way ANOVA followed by the Tukey's multiple comparison test s with GraphPad 6.0.

\section{Declarations}

\section{Funding}

This work was supported by the National Natural Science Foundation of China (grant number 82073741).

\section{Competing interests}

The authors declare no competing interests.

\section{Author contributions}

Conceptualization, L. B., D. Y.; Methodology, L. B., D. C. J. and G. Y. Z.; Investigation, L. B. and Y. Z.; Writing, L. B., and D. Y.; Supervision, D. Y.; Funding Acquisition, L. B.

\section{Data availability}

Extended data is available for this paper at https://doi.org/XX.

\section{References}

1. Leitner, D. R., et al. Obesity and type 2 diabetes: Two diseases with a need for combined treatment strategies-EASO can lead the way. Obes. Facts. 10, 483-492 (2017).

2. Maruvada, P., Leone, V., Kaplan, L. M. \& Chang, E. B. The human microbiome and obesity: moving beyond associations. Cell Host Microbe. 22, 589-599 (2017).

3. Clemente, J. C., Ursell, L. K., Parfrey, L. W. \& Knight R. The impact of the gut microbiota on human health: An integrative view. Cell 148, 1258-1270 (2012).

4. Cani, P. D., Osto, M., Geurts, L. \& Everard, A. Involvement of gut microbiota in the development of lowgrade inflammation and type 2 diabetes associated with obesity. Gut Microbes. 3, 279-288 (2012).

5. Krautkramer, K. A., Fan, J. \& Backhed, F. Gut microbial metabolites as multi-kingdom intermediates. Nat. Rev. Microbiol. 19, 77-94 (2021).

6. Koh, A., et al. Microbially produced imidazole propionate impairs insulin signaling through mTORC1. Cell 175, 947-961.e17 (2018). 
7. Sun, L. L., et al. Gut microbiota and intestinal FXR mediate the clinical benefits of metformin. Nat. Med. 24, 1919-1929 (2018).

8. Limon, J. J., Skalski, J. H. \& Underhill, D. M. Commensal fungi in health and disease. Cell Host Microbe. 22, 156-165 (2018).

9. Iliev, I. D. \& Leonardi, I. Fungal dysbiosis: immunity and interactions at mucosal barriers. Nat. Rev. Immunol. 17, 625-646 (2017).

10. Maurya, V., et al. Sensitization to Aspergillus antigens and occurrence of allergic bronchopulmonary aspergillosis in patients with asthma. Chest 127, 1252-1259 (2005).

11. Moyes, D. L. \& Naglik, J. R. The mycobiome: influencing IBD severity. Cell Host Microbe. 11, 551-552 (2012).

12. Yang, A. M., et al. Intestinal fungi contribute to development of alcoholic liver disease. J. Clin. Invest. 127, 2829-2841 (2017).

13. Aykut, B., et al. The fungal mycobiome promotes pancreatic oncogenesis via activation of MBL. Nature 574, 264-267 (2019).

14. Chu, H. K., et al. The Candida albicans exotoxin Candidalysin promotes alcohol-associated liver disease. J, Hepatol. 72, 391-400 (2020).

15. Lemke, A., Kiderlen, A. F. \& Kayser, O. Amphotericin B. Appl. Microbiol. Biotechnol. 68, 151-162 (2005).

16. Ouellette, M., Drummelsmith, J. \& Papadopoulou, B. Leishmaniasis: Drugs in the clinic, resistance and new developments. Drug Resist. Updat. 7, 257-266 (2004).

17. Steven, E. K., Rebecca, L. H. \& Kristina, M. U. Mechanisms linking obesity to insulin resistance and type 2 diabetes. Nature 444, 840-846 (2006).

18. Annie, S. V., et al. Candida albicans Is an Immunogen for Anti-Saccharomyces cerevisiae Antibody Markers of Crohn's Disease. Gastroenterol. 130, 1764-1775 (2006).

19. Yamahiro, A., Lau, K. H., Peaper, D. R. \& Villanueva, M. Meningitis caused by Candida Dubliniensis in a patient with cirrhosis: a case report and review of the literature. Mycopathologia 181, 589-593 (2016).

20. Theel, E. S. \& Doern, C. D. $\beta$-D-glucan testing is important for diagnosis of invasive fungal infections. J. Clin. Microbiol. 51, 3478-3483 (2013).

21. Iliev1, I. D., et al. Interactions between commensal fungi and the C-Type lectin receptor dectin-1 influence Colitis. Science 336, 1314-1317 (2012). 
22. Sun, S. S., et al. An insoluble polysaccharide from the sclerotium of Poria cocos improves hyperglycemia, hyperlipidemia and hepatic steatosis in ob/ob mice via modulation of gut microbiota. Chin. J. Nat. Med. 17, 3-14 (2019).

23. Sun, S. S., et al. Therapeutic manipulation of gut microbiota by polysaccharides of Wolfiporia cocos reveals the contribution of the gut fungi-induced PGE2 to alcoholic hepatic steatosis. Gut Microbes. 12, e1830693 (2020).

24. Kono, $\mathrm{H}$., et al. NMR spectroscopic structural characterization of a water-soluble $\beta-(1 \rightarrow 3,1 \rightarrow 6)$-glucan from Aureobasidium pullulans. Carbohyd. Polym. 174, 876-886 (2017).

25. del Fresno, C., et al. Interferon-beta production via Dectin-1-Syk-IRF5 signaling in dendritic cells is crucial for immunity to C. albicans. Immunity. 38, 1176-1186 (2013).

26. Weiss, M., et al. IRF5 is a specific marker of inflammatory macrophages in vivo. Mediat. Inflamm. 245804 (2013).

27. Richard, M. L. \& Sokol, H. The gut mycobiota: insights into analysis, environmental interactions and role in gastrointestinal diseases. Nat. Rev. Gastroenterol. Hepatol. 16, 331-345 (2019).

28. Ley, R. E., Turnbaugh, P. J., Klein, S. \& Gordon, J. I. Microbial ecology: human gut microbes associated with obesity. Nature 444, 1022-1023 (2006).

29. Koliada, A., et al. Association between body mass index and Firmicutes/Bacteroidetes ratio in an adult Ukrainian population. BMC Microbiol. 17, 120 (2017).

30. Chang, C. J., et al. Ganoderma lucidum reduces obesity in mice by modulating the composition of the gut microbiota. Nat. Commun. 6, 7489 (2015).

31. Cani, P. D. \& de Vos, W. M. Next-generation beneficial microbes: the case of Akkermansia muciniphila. Front. Microbiol. 8, 1765 (2017).

32. Herriott, M. M. \& Noverr, M. C. Importance of Candida-bacterial polymicrobial biofilms in disease. Trends Microbiol. 19, 557-563 (2011).

33. Manco, M., Putignani, L., Bottazzo, G. F. Gut microbiota, lipopolysaccharides, and innate immunity in the pathogenesis of obesity and cardiovascular risk. Endocr. Rev. 31, 817-844 (2010).

34. Zhang, D., et al. The mycobiota of the human body: a spark can start a prairie fire. Gut microbes. 11, 655-679 (2020).

35. Standaert-Vitse, A., et al. Candida albicans colonization and ASCA in familial Crohn's disease. Am. J. Gastroenterol. 104, 1745-1753 (2009). 
36. Aykut, B., et al. The fungal mycobiome promotes pancreatic oncogenesis via activation of MBL. Nature 574, 264-267 (2019).

37. Shao, T. Y., et al. Commensal Candida albicans Positively Calibrates Systemic Th17 Immunological Responses. Cell Host Microbe. 25, 404-17.e6 (2019).

38. Mehta, N.N., et al. Experimental endotoxemia induces adipose inflammation and insulin resistance in humans. Diabetes 59, 172-181 (2010).

39. Everard, A., et al. Cross-talk between Akkermansia muciniphila and intestinal epithelium controls dietinduced obesity. P. Natl. Acad. Sci. USA. 110, 9066-9071 (2013).

40. Cani, P. D., et al. Changes in gut microbiota control metabolic endotoxemia-induced inflammation in high-fat diet-induced obesity and diabetes in mice. Diabetes 57, 1470-1481 (2008).

41. Cruz-Aguliar, R. M., et al. An Open-Labeled study on fecal microbiota transfer in irritable bowel syndrome patients reveals improvement in abdominal pain associated with the relative abundance of Akkermansia Muciniphila. Digestion 100, 127-138 (2019).

42. Dingemanse, C., et al. Akkermansia muciniphila and Helicobacter typhlonius modulate intestinal tumor development in mice. Carcinogenesis 36, 1388-1396 (2015).

43. Olson, C. A., et al. The gut microbiota mediates the anti-seizure effects of the Ketogenic diet. Cell 173, 1728-1741 (2018).

44. Wang, $\mathrm{K}$., et al. A novel class of alpha-glucosidase and HMG-CoA reductase inhibitors from Ganoderma leucocontextum and the antidiabetic properties of ganomycin I in KK-Ay mice. Eur. J. Med. Chem. 127, 1035-1046 (2017).

45. Hamad, l., et al. Culturomics and amplicon-based metagenomic approaches for the study of fungal population in human gut microbiota. Sci. Rep. 7, 16788 (2017).

46. Saxena. A, McElhaney-Feser. G. E., \& Cihlar, R. L. Mannan composition of the hyphal form of two relatively avirulent mutants of Candida albicans. Infect. Immun. 58, 2061-2066 (1990).

\section{Figures}



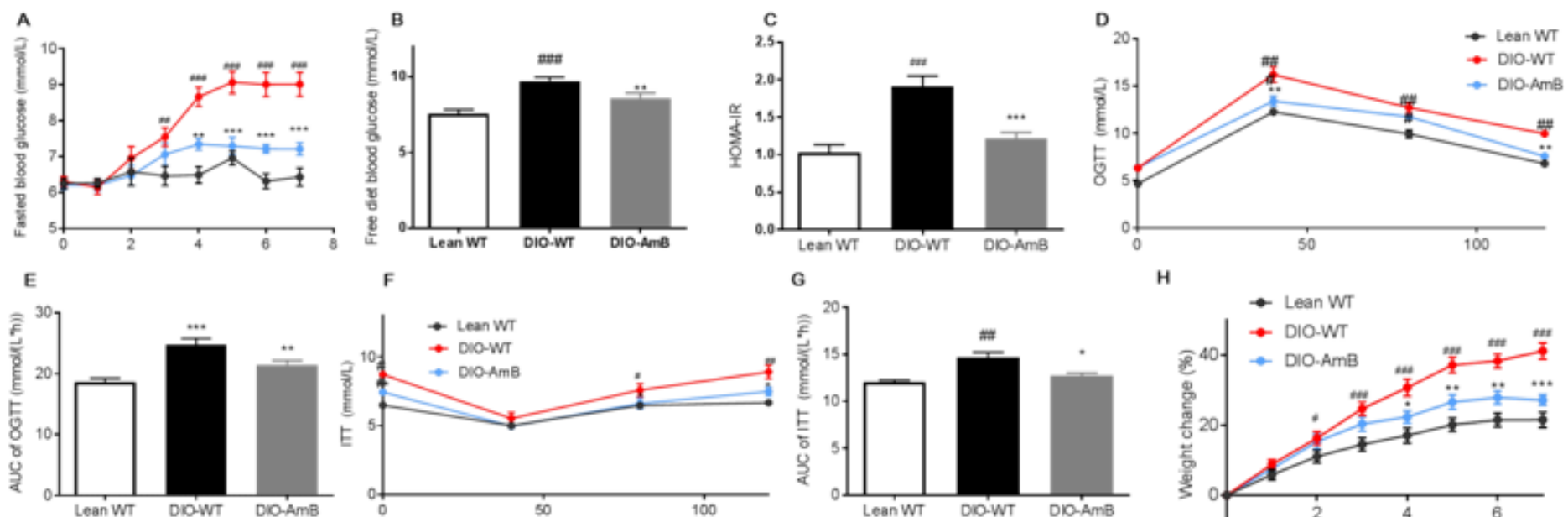

F

G

H
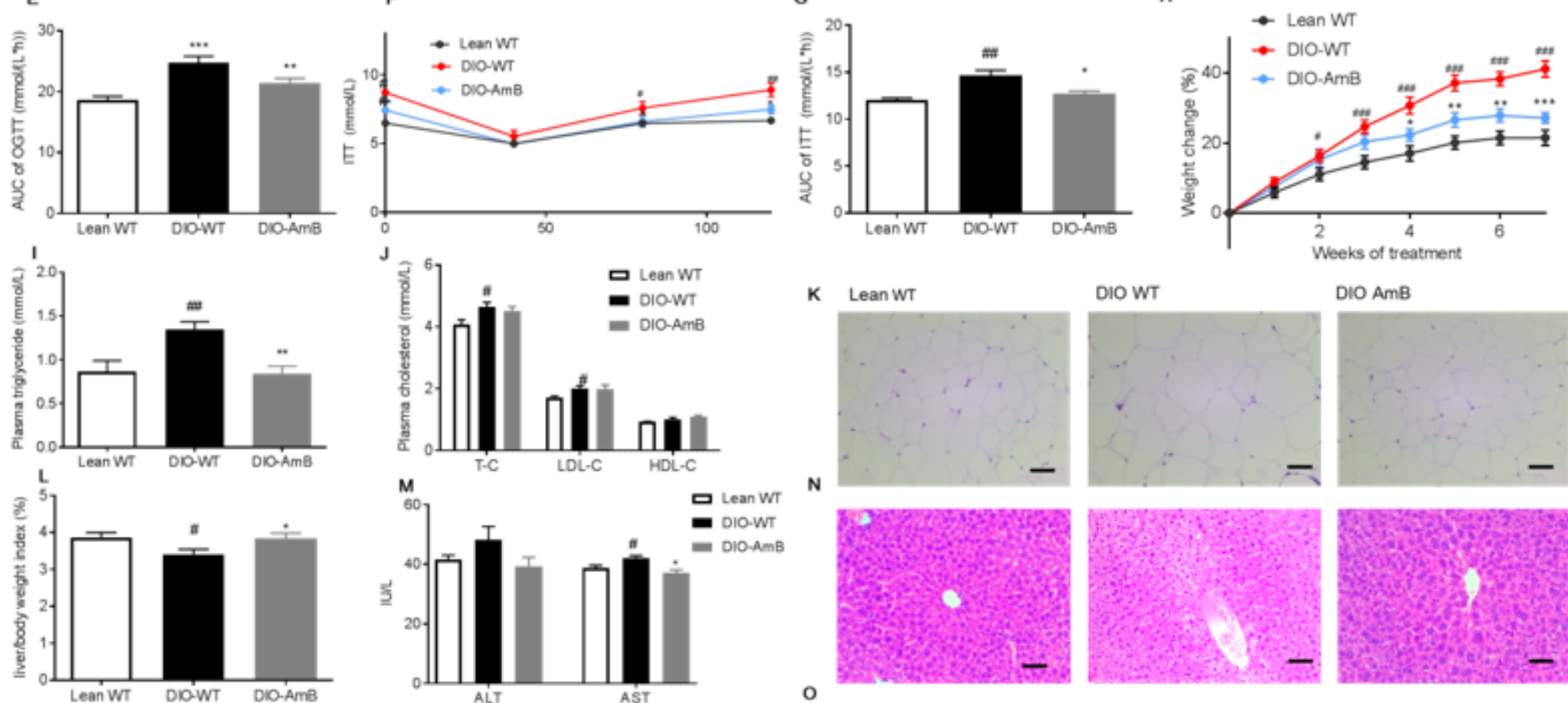

DIO WT

DIO AmB
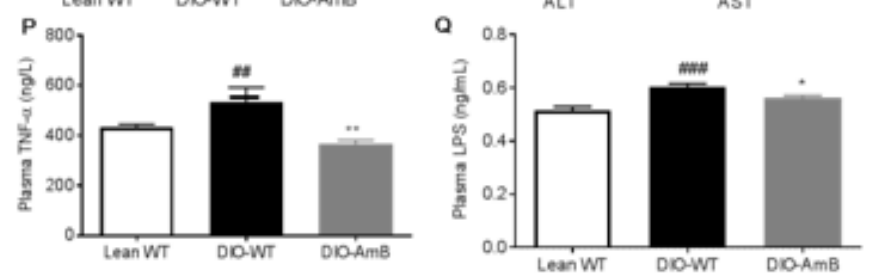

o
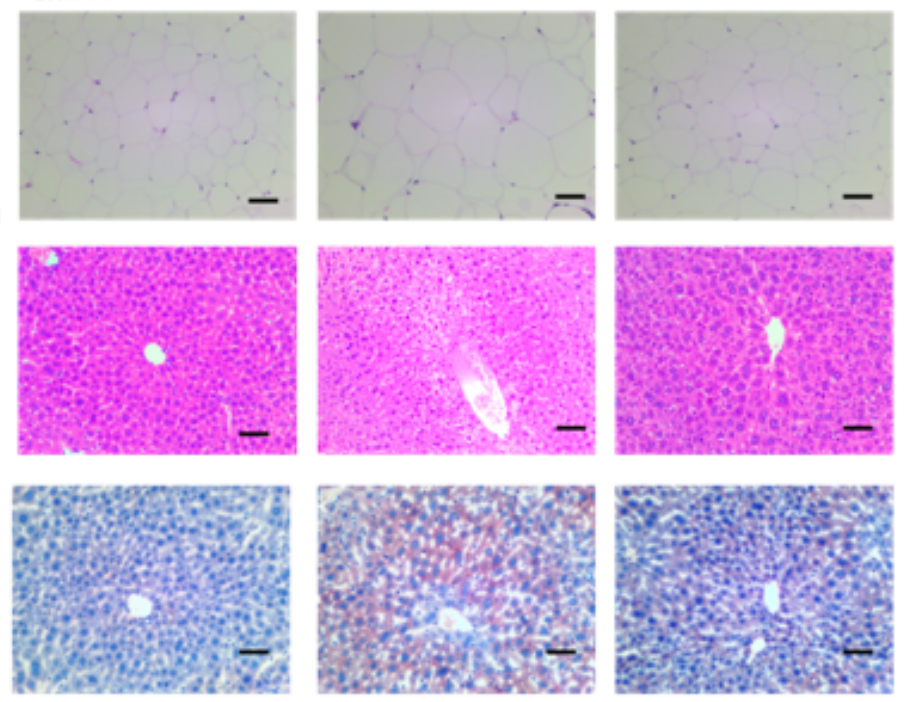

Figure 1

Amphotericin B treatment alleviated glucose metabolism related disorders in HFD-fed mice. (A) Fasted blood glucose, (B) Free blood glucose ( $6^{\text {th }}$ week), (C) HOMA-IR, (D) OGTT and (E) AUC, (F) ITT and (G) AUC, (H) Weight change, (I) Plasma triglyceride, (J) Plasma total cholesterol, LDL-C and HDL-C, (K) representative $\mathrm{H}$ \& E-stain pictures of WAT tissue, (L) liver/body weight ratio, (M) plasma alanine trasaminase (ALT) and aspartate transminase (AST), (N) and (O) representative $\mathrm{H}$ \& E-stain and oil-red stain pictures of liver tissue, (P) Plasma TNF-a, (Q) Plasma LPS. Data are presented as the mean \pm standard error of the mean (SEM); $N=8-10$ mice per group. Statistical analysis was done using oneway ANOVA followed by the Tukey post hoc test. $* \mathrm{P}<0.05 ;{ }^{*} \mathrm{P}<0.01$; *** $\mathrm{P}<0.001$. 


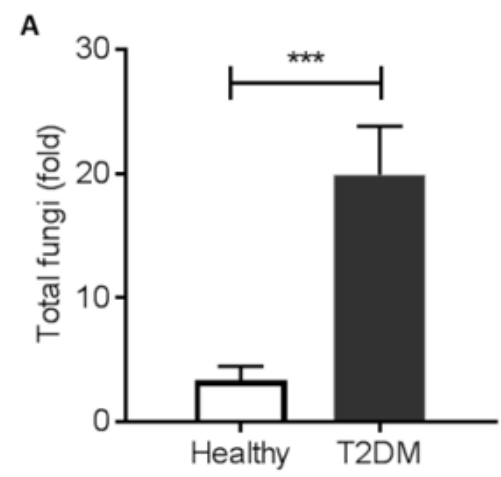

D


B

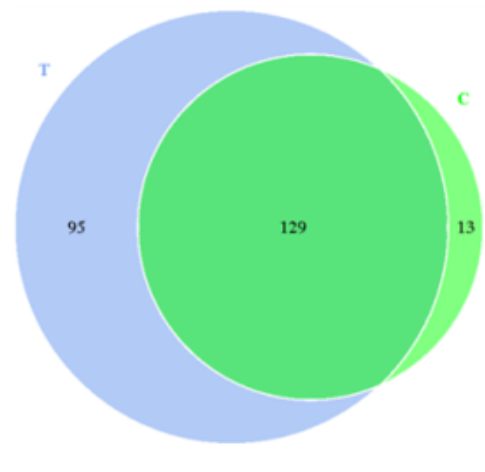

E
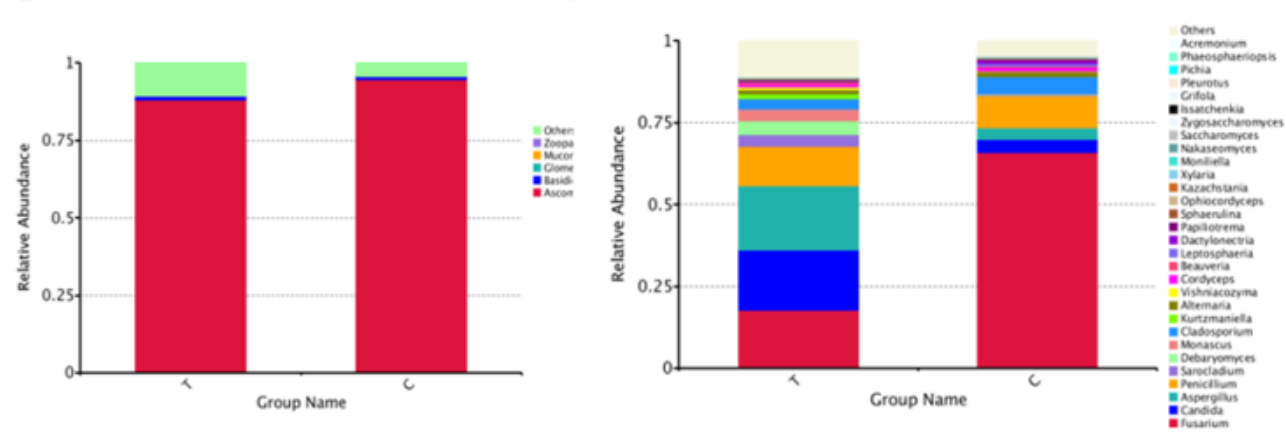

C

$\mathbf{F}$
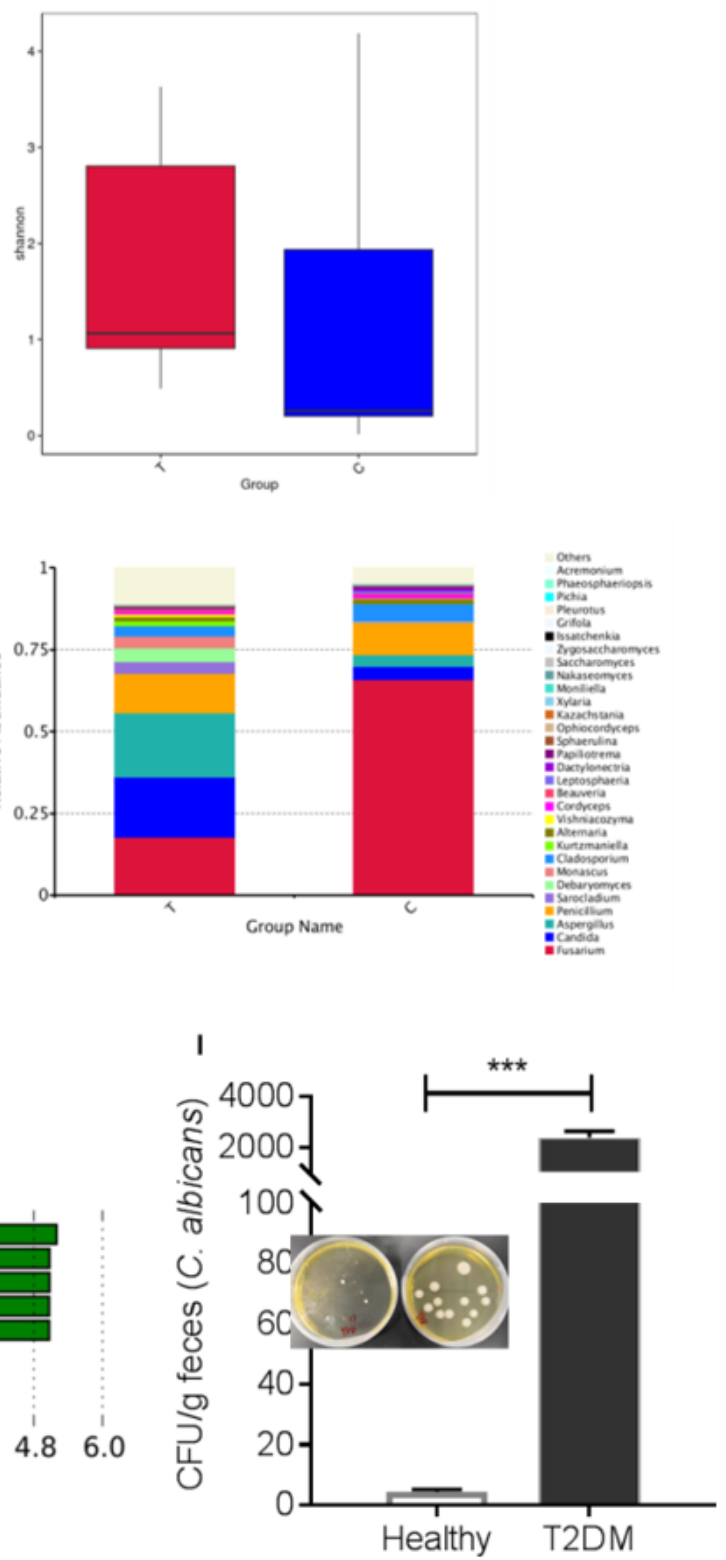

Figure 2

Gut mycobiome (fungal community) alternations in T2D patients. (A) Total fungi in feces were assessed by qPCR, (B) Venn diagram based on genera, (C) Shannon index, (D) Unweighted uniFrac-based principal coordinates analysis, Change in fungi at the phylum (E) and genus (F) levels, $(G)$ Cladogram generated from LEfSe analysis showing the relationship between taxon, $(H)$ Linear discriminant analysis (LDA) scores derived from LEfSe analysis, (I) CFUs of $C$. albicans in fecal samples. T: T2D patients, C: healthy volunteers. 

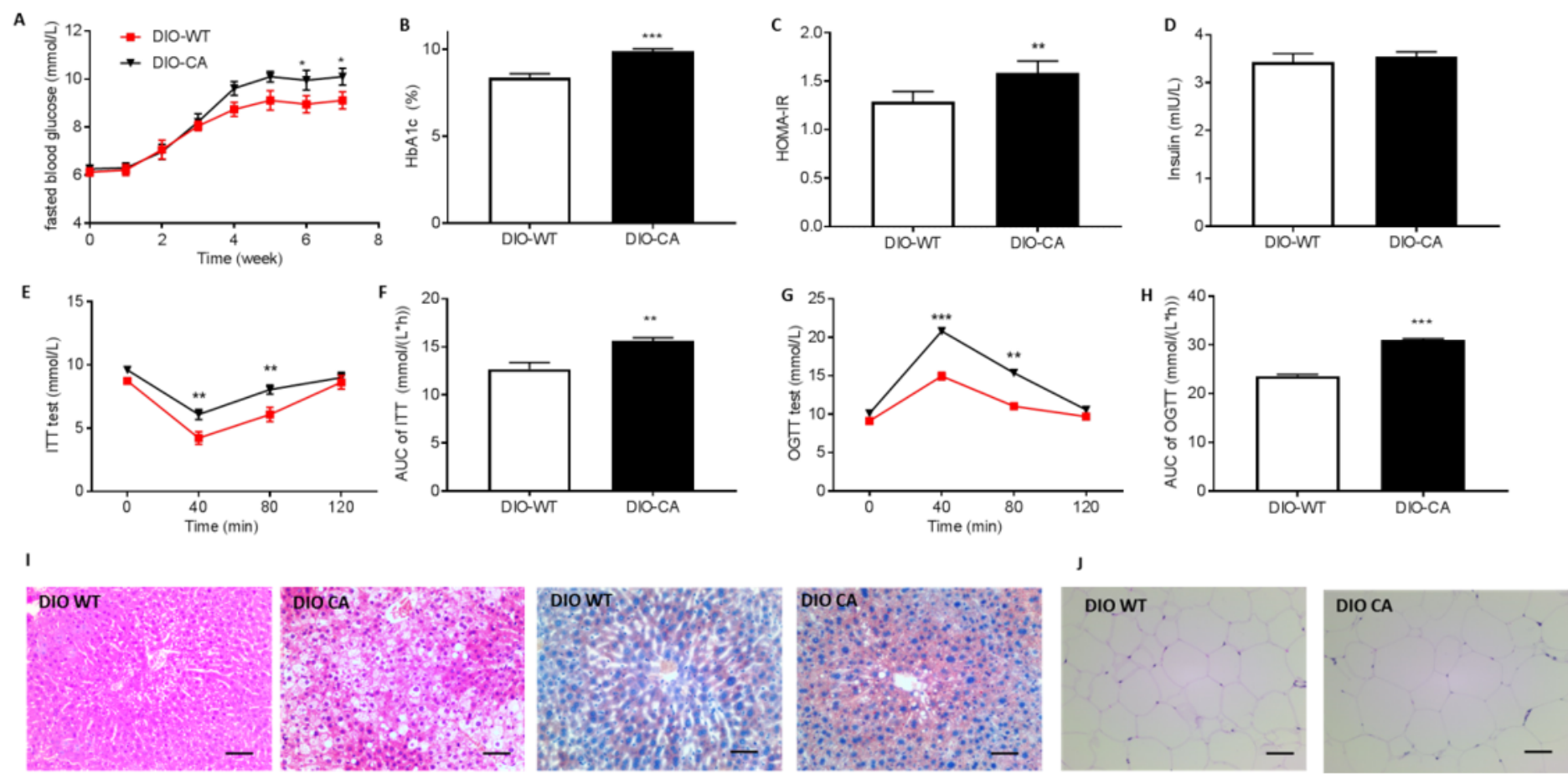

Figure 3

Effects of $C$. albicans strains isolated from T2DM patients on the induction of insulin resistance. (A) fasted blood glucose; (B) HbA1c in HFD-feeding mice; (C) HOMA-IR; (D) insulin level; (E) ITT and (F) AUC on the 29th day; $(\mathrm{G})$ OGTT and $(\mathrm{H})$ AUC on the 36th day of treatment in HFD-feeding mice; (I) representative images of $\mathrm{H}$ \& E-stain, oil-red stain of liver tissue, and $(\mathrm{J})$ representative images of $\mathrm{H}$ \& Estain of adipose tissue ( $N=5$ mice per group). Data are presented as the mean \pm standard error of the mean (SEM); $\mathrm{N}=8-10$ mice per group. Statistical analysis was done using oneway ANOVA followed by the Tukey post hoc test. $* \mathrm{P}<0.05 ; * * \mathrm{P}<0.01 ; * * * \mathrm{P}<0.001$.

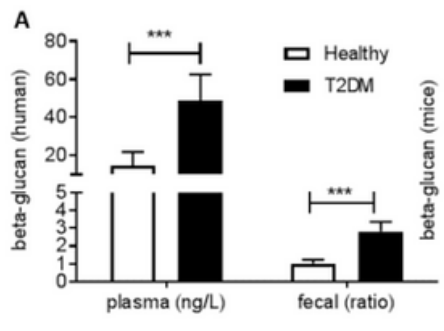

E

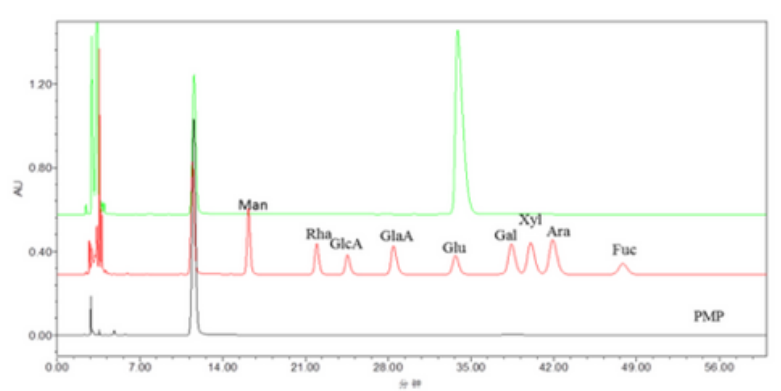

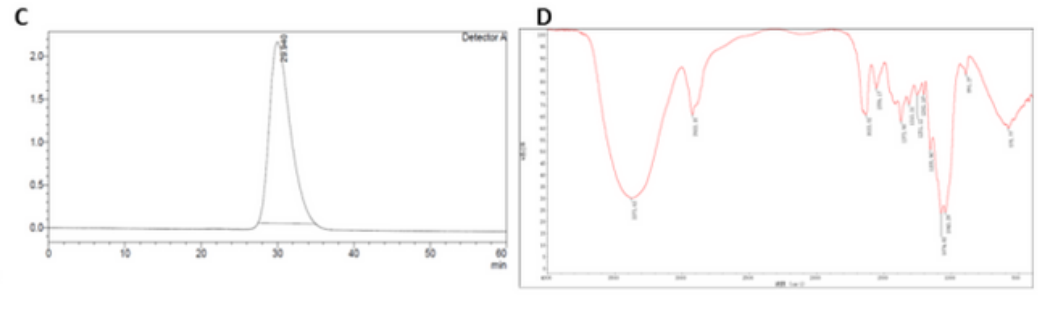

$\mathbf{F}$

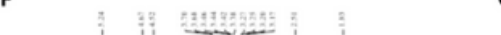

Figure 4 
The characterization of 1,3- $\beta$-glucan isolated from $C$. albicans. (A) 1,3- $\beta$-glucans content of plasma and fecal in human (A) and mice (B), (C-G) HPGPC, FT-IR and NMR in DMSO- $d_{6}$ spectrum of 1,3- $\beta$-glucan isolated from $C$. albicans.
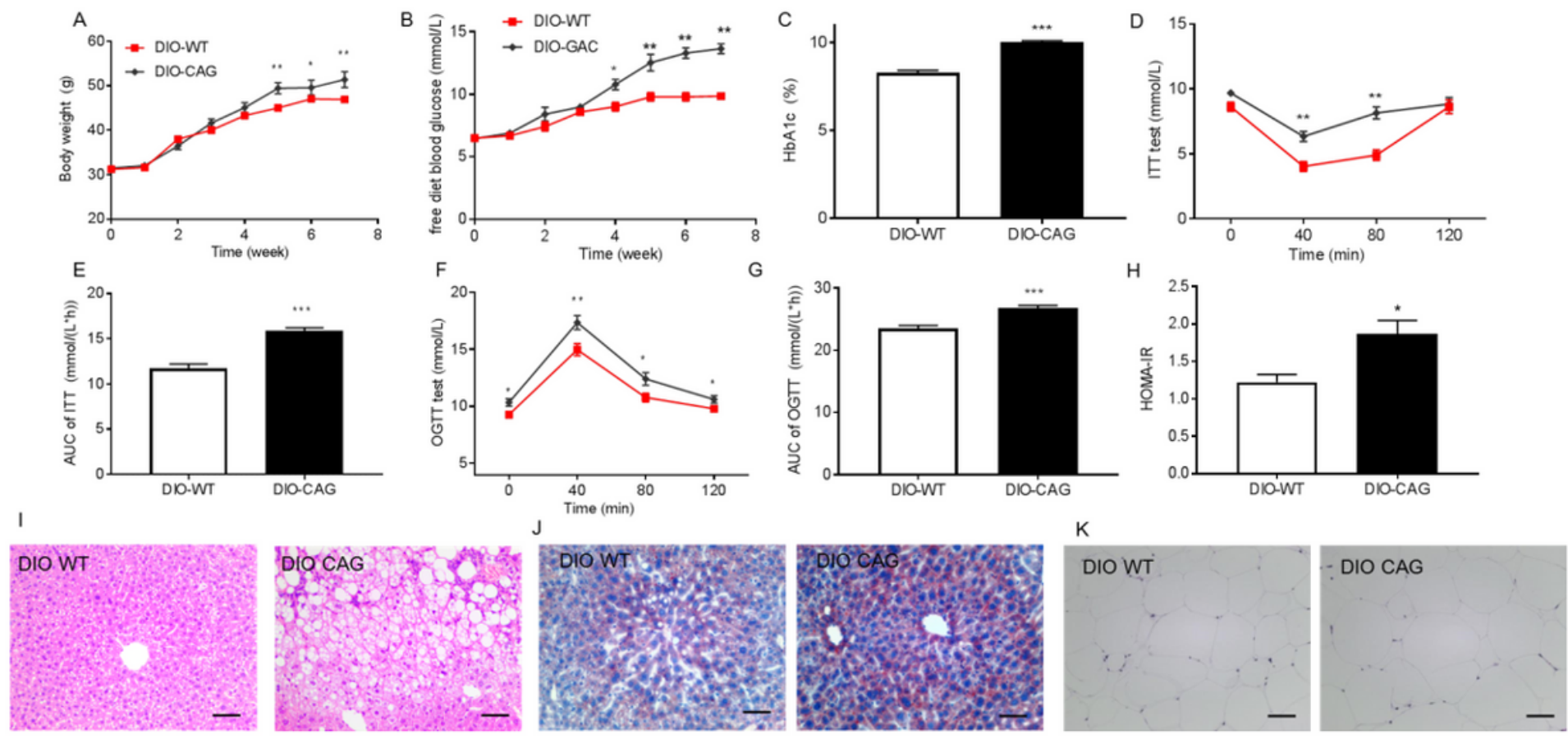

Figure 5

Feeding with 1,3- $\beta$-Glucan isolated from $C$. albicans (CAG) promoted insulin resistance. (A) and (B) sequential monitoring of blood glucose after $4 \mathrm{~h}$ fasting or free diet; (C) HbA1c in HFD-fed mice; (D) ITT and (E) AUC on the 29th day; (F) OGTT and (G) AUC on the 36th day of treatment in HFD-fed mice; $(\mathrm{H})$ HOMA-IR; (I-J) representative images of H \& E-stain, oil-red stain of liver tissue, and $(\mathrm{K})$ representative images of $\mathrm{H} \& \mathrm{E}$-stain of adipose tissue $(\mathrm{N}=5$ mice per group). Data are presented as the mean \pm standard error of the mean (SEM); $N=8-10$ mice per group. Statistical analysis was done using oneway ANOVA followed by the Tukey post hoc test. $* \mathrm{P}<0.05$; $* * \mathrm{P}<0.01$; $* * * \mathrm{P}<0.001$. 

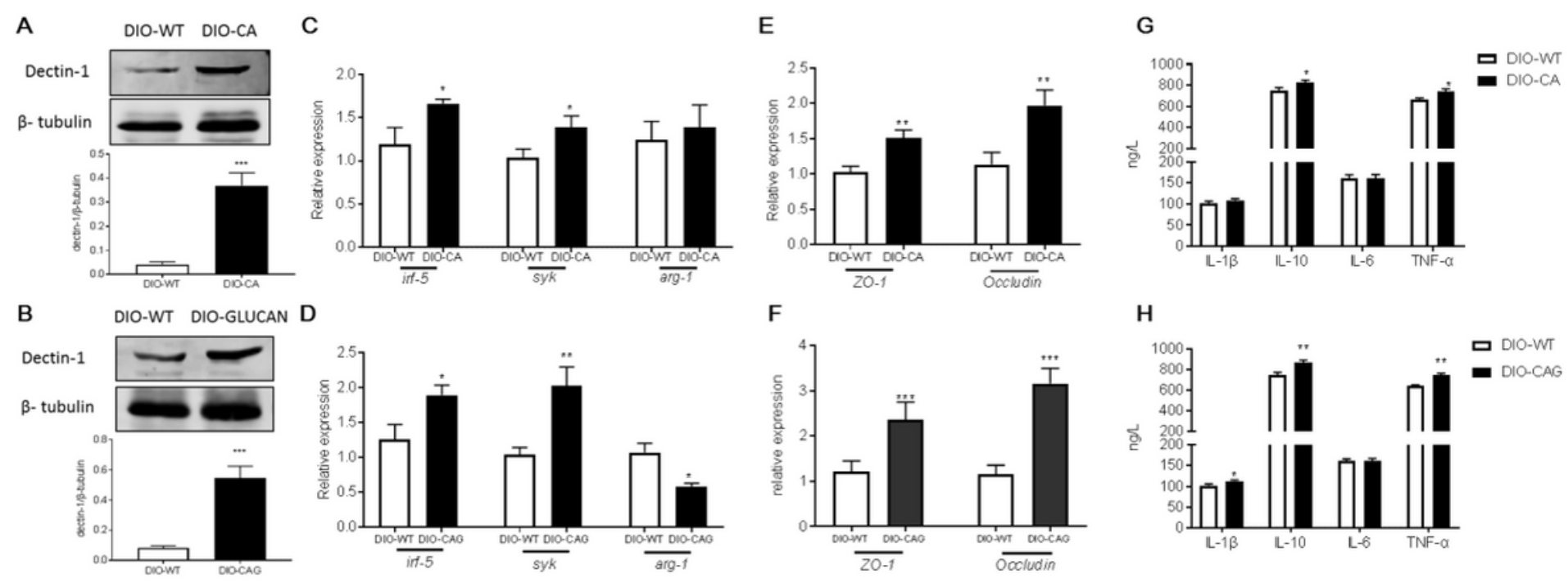

Figure 6

CAG induce insulin resistance though the activation of dectin-1 pathway. (A) and (B) The colon dectin-1 level of the $C$. albicans-treated or CAG-treated mice; (C) and (D) The expression of irf-5, syk and arg- 1 in colon; (E) and (F) The expression of ZO-1 and occludin in colon; $(\mathrm{G})$ and $(\mathrm{H})$ The serum levels of IL-1 $\beta$, IL10, IL- 6 and TNF- $a$. Data are presented as the mean \pm standard error of the mean (SEM); $N=5-8$ mice per group. Statistical analysis was done using oneway ANOVA followed by the Tukey post hoc test. $* \mathrm{P}<$ $0.05 ; * * \mathrm{P}<0.01 ; * * * \mathrm{P}<0.001$. 

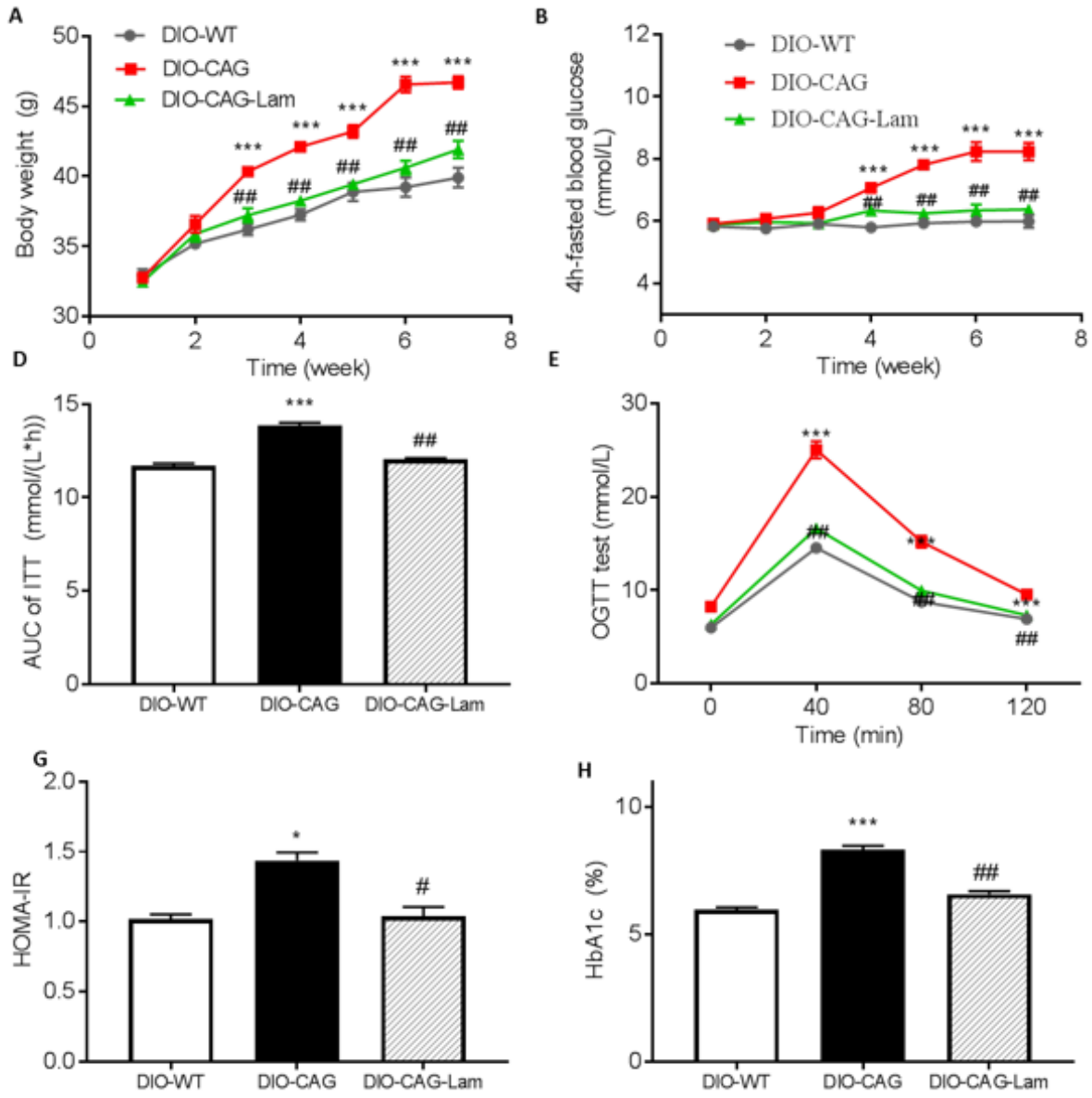

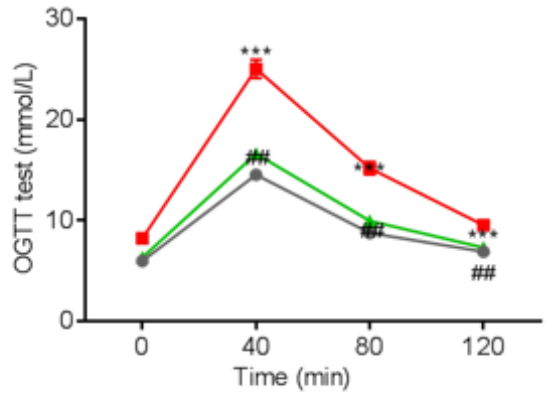

H

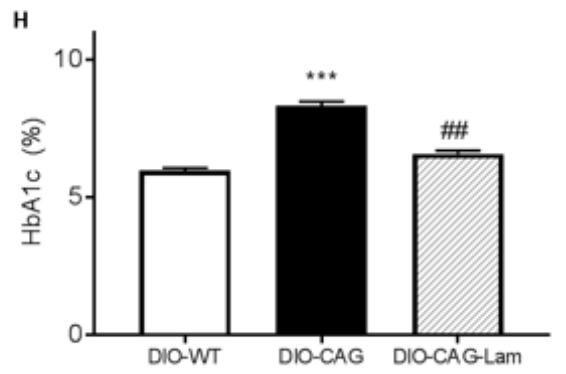

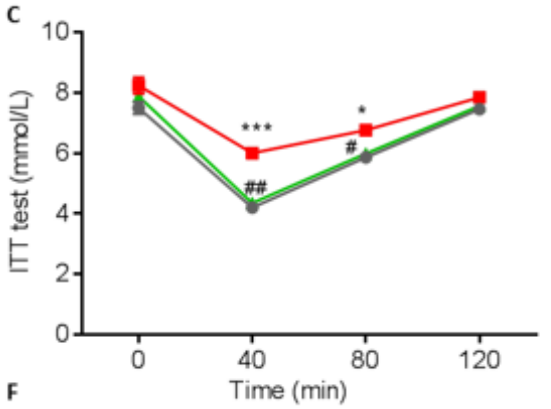
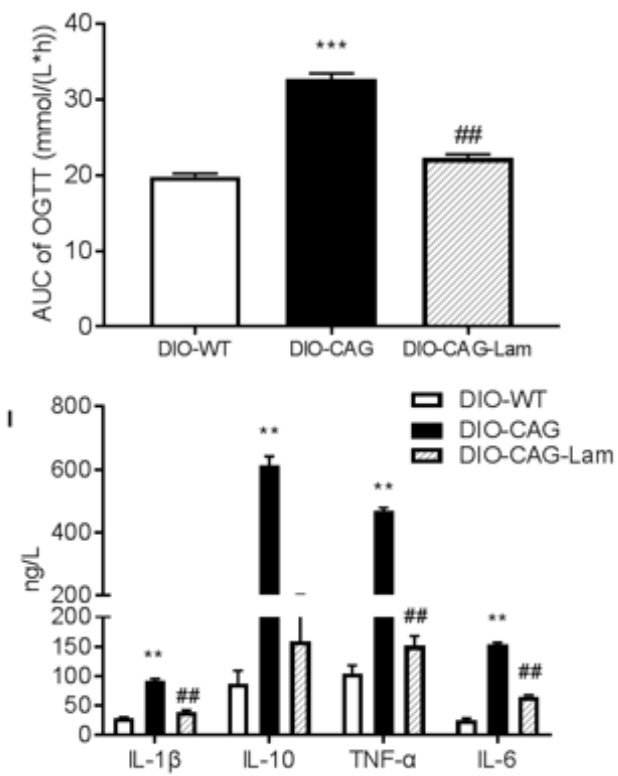

Figure 7

Dectin-1 Antagonist Laminarin treatment reversed the deteriorated effect of CAG on HFD-treated mice. (A) Body Weight, (B) Fasted blood glucose, (C) ITT and (D) AUC on the 31th day, (E) OGTT and (F) AUC on the 34th day, (G) HOMA-IR, (H) HbA1c, (I) The serum levels of IL-1 $\beta$, IL-10, IL-6 and TNF-a. Data are presented as the mean \pm standard error of the mean (SEM); $N=10$ mice per group. Statistical analysis was done using oneway ANOVA followed by the Tukey post hoc test. ${ }^{*} \mathrm{P}<0.05$; ${ }^{* * *} \mathrm{P}<0.001$ compared with DIOWT group, and ${ }^{\#} \mathrm{P}<0.05$; ${ }^{\# \#} \mathrm{P}<0.001$ compared with DIO-CAG group. 
A

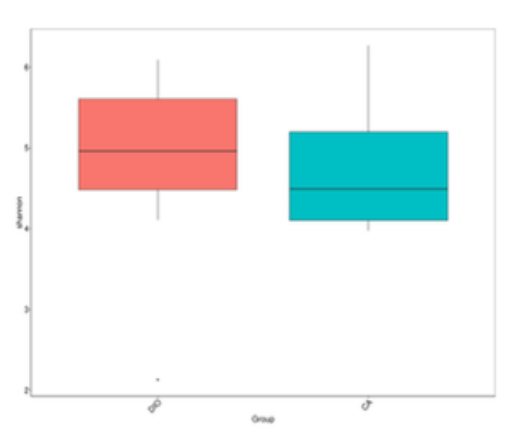

C

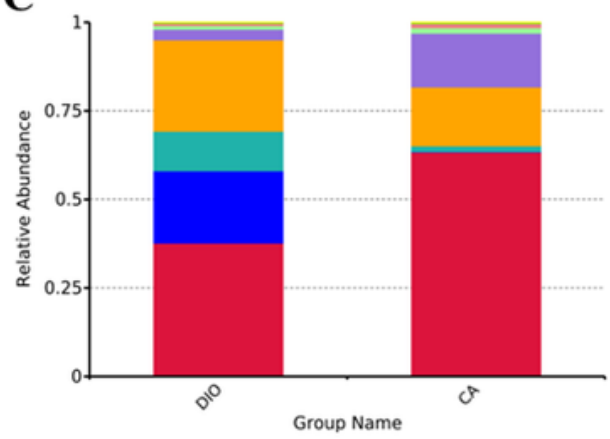

$\mathbf{E}$

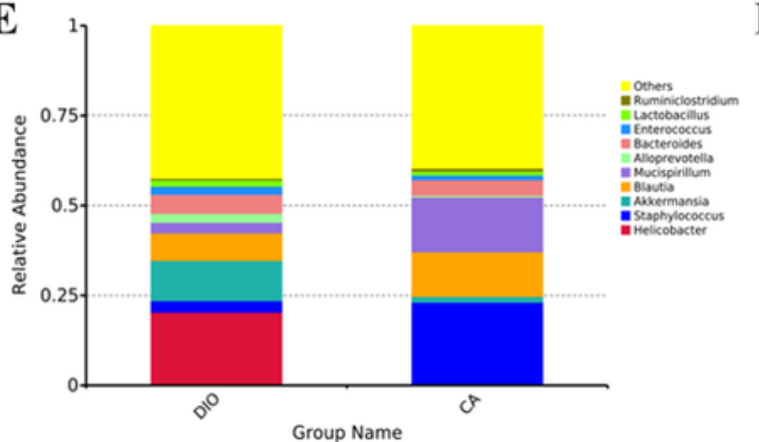

G

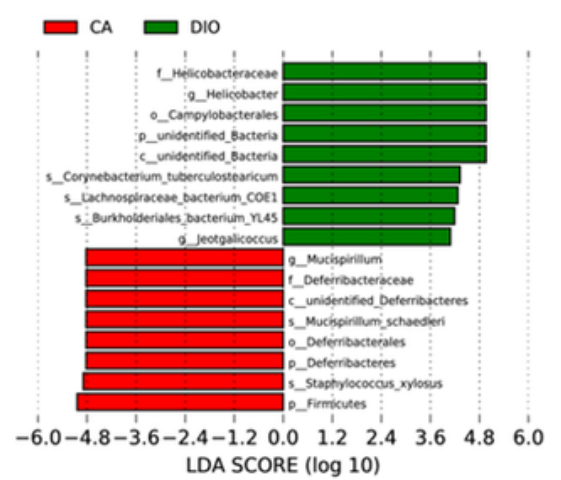

PCoA plot

D
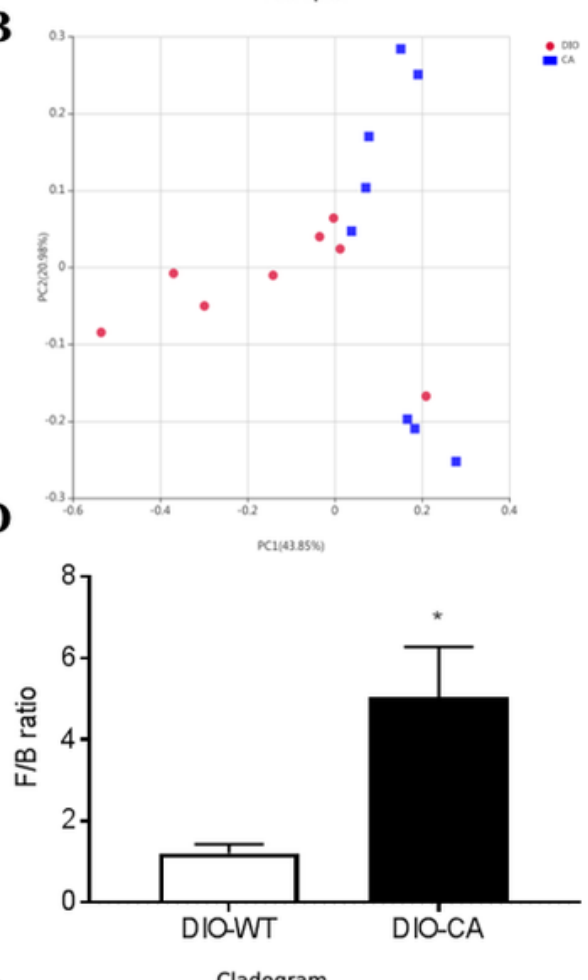

$\mathbf{F}$

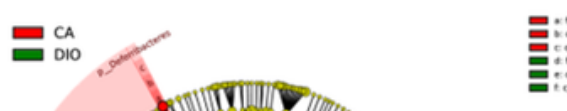

를:

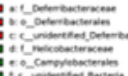

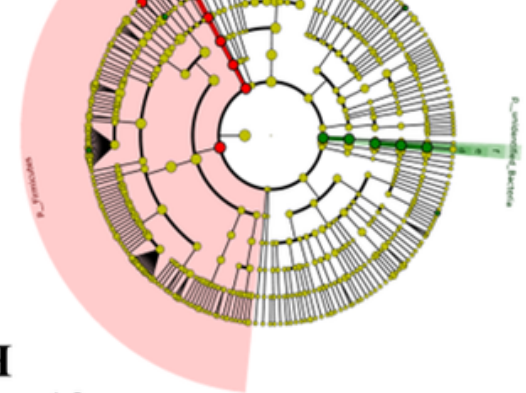

$\mathbf{H}$

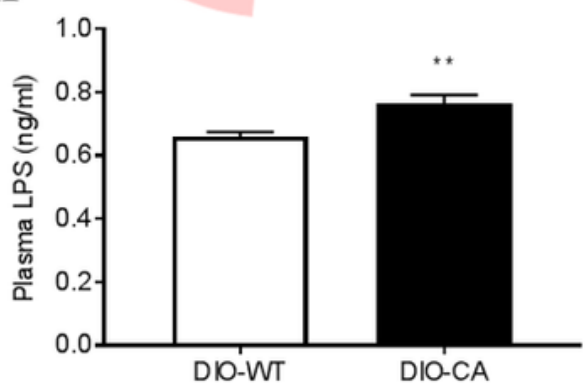

\section{Figure 8}

Intestinal fungi $\boldsymbol{C}$. albicans changed the gut microbiota structure in HFD-fed mice. (A) Shannon index, (B) Principal coordinate analysis (PCOA) of all samples by weighted UniFrac distance, (C) Change in Phylum with $C$. albicans treatment, (D) Firmicutes/Bacteroidetes radio, (E) Change in genus with $C$. albicans treatment, $(F)$ Cladogram generated from LEfSe analysis showing the relationship between taxon, $(G)$ Linear discriminat analysis (LDA) scores derived from LEfSe analysis, (H) Plasma LPS level. 


\section{Supplementary Files}

This is a list of supplementary files associated with this preprint. Click to download.

- SI0211.docx 